\title{
High-speed visible light communication based on a III-nitride series-biased micro-LED array
}

\author{
Enyuan Xie, Xiangyu He, Mohamed Sufyan Islim, Graduate Student Member, IEEE, Ardimas Andi Purwita, \\ Jonathan J. D. McKendry, Erdan Gu, Member, IEEE, Harald Haas, Fellow, IEEE, \\ and Martin D. Dawson, Fellow, IEEE
}

\begin{abstract}
Visible light communication (VLC) using III-nitride light-emitting diodes (LEDs) offers many advantages such as license-free operation, high spatial diversity and innate security. In particular, micro-LEDs ( $\mu$ LEDs) are strong candidates for VLC due to their high modulation bandwidths. However, the low optical power of a single $\mu$ LED is a key factor limiting VLC performance. In this work, we report an optimized series-biased $\mu$ LED array to achieve higher optical power while retaining high modulation bandwidth for high-speed VLC. An example array consisting of $3 \times 340 \mu \mathrm{m}$-in-diameter $\mu$ LED elements is presented here. At a current density of $3200 \mathrm{~A} / \mathrm{cm}^{2}$ in direct-current operation, the optical power and small signal 6-dB electrical modulation bandwidth of a blue-emitting series-biased $\mu$ LED array are over $18.0 \mathrm{~mW}$ and $285 \mathrm{MHz}$, respectively. The data transmission capabilities of this $\mu$ LED array are demonstrated by using onoff-keying, pulse-amplitude modulation, and orthogonal frequency division multiplexing modulation formats over free space with the error-free data transmission rates of $1.95,2.37$ and 4.81 Gbps, respectively.
\end{abstract}

Index Terms-Visible light communication, Series-biased micro-LED array.

\section{INTRODUCTION}

V ISIBLE light communication (VLC), which can be integrated into pre-existing lighting infrastructure, is an attractive technology. It opens up the wider licence-free, visible region of the electromagnetic spectrum for wireless communications and, thus, offers a promising alternative to the bandwidth limitation of the radio frequency (RF) spectrum. Furthermore, the spectral efficiency per unit area is also enhanced by VLC, which leads to high spatial diversity and innate security [1]. Light-emitting diodes (LEDs) based on III-nitride materials are very promising light sources for VLC systems due to their capabilities to efficiently generate light covering the whole visible spectrum and be modulated

Enyuan Xie, Xiangyu He, Jonathan J. D. McKendry, Erdan $\mathrm{Gu}$ and Martin D. Dawson are with Institute of Photonics, Department of Physics, University of Strathclyde, Glasgow, G1 1RD, UK (e-mail: enyuan.xie@strath.ac.uk; xiangyu.he@strath.ac.uk; jonathan.mckendry@strath.ac.uk; erdan.gu@strath.ac.uk; m.dawson@strath.ac.uk).

Mohamed Sufyan Islim, Ardimas Andi Purwita and Harald Haas are with LiFi R\&D Centre, the University of Edinburgh, Institute for Digita Communications, Kings Buildings, Mayfield Road, Edinburgh, EH9 3JL, UK (e-mail: M.islim@ed.ac.uk; A.Purwita@ed.ac.uk; H.Haas@ed.ac.uk).

Enyuan Xie, Xiangyu He and Mohamed Sufyan Islim contribute equally to this work.

Manuscript received April 19, 2005; revised August 26, 2015. This research was supported by Engineering and Physical Sciences Research Council (EPSRC) grant EP/K00042X/1 (UP-VLC). Data is available online at xxxxxxxxx. (Corresponding auther: Enyuan Xie and Erdan Gu) faster than conventional incandescent or fluorescent light sources. The typical 6-dB electrical modulation bandwidth (corresponding to $3-\mathrm{dB}$ optical modulation bandwidth) of commercial III-nitride LEDs is in the range of $10-20 \mathrm{MHz}$ [2], which is limited by the large resistance-capacitance (RC) time constant of the p-n junction in conventional broad-area LEDs. Through different modulation schemes such as parallel data transmission and equalization, the VLC data transmission rates based on these LEDs are in excess of 1 Gbps [3], [4]. A recorded data transmission rate up to 2.32 Gbps using commercial III-nitride LEDs has been reported recently [5].

In order to further increase the data transmission rate of VLC systems based on III-nitride LED light sources, much effort has been made to optimize the LED epitaxial structure and device configuration [6], [7]. In recent years, microLED ( $\mu$ LED) emitters, which have lateral dimensions of less than $100 \mu \mathrm{m}$, have emerged to enable high-speed VLC applications [8]. The smaller LED junction area of $\mu$ LEDs leads to the small RC time constant and high operation current density [9]. These novel characteristics increase the 6-dB electrical bandwidth of $\mu$ LEDs up to a few hundred MHz [10] and, thus, make them strong candidates for VLC systems. In early works, by using on-off keying (OOK) and orthogonal frequency division multiplexing (OFDM) modulation schemes, data transmission rates up to $1.7 \mathrm{Gbps}$ [10] and $7.9 \mathrm{Gbps}$ [11] have been achieved at the forward error correction (FEC) floor of $3.8 \times 10^{-3}$, applied to single $\mu$ LEDs. However, the low optical power produced from a single $\mu$ LED is considered as a main factor limiting wider use of this technology in VLC applications. According to the Shannon-Hartley theorem [12], the maximum data transmission rate, $D$, over a communication channel can be determined by $D=B \log _{2}(1+S / N)$, where $B$ is the channel bandwidth; $S$ is the average received signal power over the bandwidth; $N$ is the average power of the noise assumed to be white Gaussian; and $S / N$ is the electrical signalto-noise ratio (SNR). This theorem indicates that low signal power would result in a low SNR and, consequently, low data transmission rate over limited transmission distances. In [13], we employed a $\mu$ LED array operating in a ' ganged' fashion to increase the optical power and, thus, signal power produced for VLC systems. However, in order to retain the high modulation bandwidth of this $\mu$ LED array, each $\mu$ LED element needed to be individually addressed by its own anode or cathode. This leads to a complex design, fabrication and integration process for both $\mu \mathrm{LED}$ array and driver circuit. Therefore, it is important to develop integrated $\mu$ LED arrays with high optical 
power, high modulation bandwidth and a simple addressing scheme.

The series-biased LED arrays, which feature a monolithically-integrated configuration of multiple series-connected LED junctions, are considered to be reliable, compact and inexpensive light sources [14], [15], [16], [17], [18]. Compared with conventional broad-area LEDs, while retaining high optical power output, these LED arrays normally have a higher turnon voltage and lower driving current under direct-current (DC) operation. Thanks to this high-voltage low-current operation mode, the series-biased LED arrays could mitigate the efficiency droop occurring in conventional III-nitride broadarea LEDs [18] and simplify the mains alternating current (AC)-to-DC driver design. Furthermore, the capacitance of the series-biased LED arrays should be theoretically smaller than the one of each individual LED element in the array due to the series-connection configuration. Thus, the series-biased LED arrays have great potential for VLC applications. For example, compared with a single LED with the same total emitting area, these arrays could be, in theory, modulated faster while producing similar optical power. On the other hand, compared with the individual LED elements in the array, while retaining the fast modulation speed, the optical power of these arrays is much higher. Recently, this hypothesis has been proved in [19], which demonstrates that, as the number of LED element-in-series increases, the modulation bandwidth and optical power increase at the same operation current density. However, to the best of our knowledge, there is as yet no work demonstrating the data rates of VLC applications based on the series-biased LED arrays.

In this paper, we report the design, fabrication, characterisation and VLC applications of III-nitride series-biased $\mu$ LED arrays. An example array consisting of $3 \times 340 \mu \mathrm{m}$-in-diameter $\mu$ LED elements fabricated from a commercial blue LED wafer on sapphire is presented here. At $450 \mathrm{~nm}$, over $18.0 \mathrm{~mW}$ optical power and $285 \mathrm{MHz}$ 6-dB electrical bandwidth are achieved at $3200 \mathrm{~A} / \mathrm{cm}^{2}$ DC operating current density. We further demonstrate the free-space VLC application of this series-biased $\mu \mathrm{LED}$ array assuming OOK, pulse-amplitude modulation (PAM) and OFDM modulation formats. The errorfree data rates of 1.95, 2.37 and 4.81 Gbps are achieved, respectively. The results shown in this work not only confirm the combined high optical power and high modulation bandwidth characteristics of series-biased $\mu$ LED arrays, but also demonstrate their great potential for VLC applications.

\section{SERIES-BIASED $\mu$ LED ARRAY}

\section{A. Design and Fabrication}

The series-biased $\mu$ LED arrays were fabricated from commercial blue III-nitride LED wafers grown on c-plane (0001) sapphire with periodically patterned surfaces. The LED epitaxial structure consists of a $3.4 \mu \mathrm{m}$-thick undoped GaN buffer layer, a $2.6 \mu \mathrm{m}$-thick n-type GaN layer, eleven periods of InGaN (2.8 nm)/GaN (13.5 nm) quantum wells (QWs) emitting at $450 \mathrm{~nm}$, a $30 \mathrm{~nm}$-thick p-type AlGaN electron blocking layer and a $160 \mathrm{~nm}$-thick p-type $\mathrm{GaN}$ topmost layer.
The series-biased $\mu$ LED array presented here contains a $3 \times 3$ layout of $\mu$ LED elements in a flip-chip configuration, each with a diameter of $40 \mu \mathrm{m}\left(1256 \mu \mathrm{m}^{2}\right.$ emitting area), on a $160 \mu \mathrm{m}$ centre-to-centre pitch. Fig. 1(a) illustrates the simplified schematic structure of the series-biased $\mu$ LED array, taking two adjacent $\mu$ LED elements as an example, to highlight element-to-element electrical interconnections. The fabrication process of this series-biased $\mu$ LED array is similar to that described in our recent work [20]. Firstly, the diskshaped $\mu \mathrm{LED}$ elements are etched down to n-type GaN layer by $\mathrm{Cl}_{2}$-based inductively coupled plasma (ICP) etching, Then, in order to fully isolate each $\mu$ LED element from both $\mathrm{p}$ and n-type $\mathrm{GaN}$ layers, $\mathrm{GaN}$ mesas, in a square shape with sides of $90 \mu \mathrm{m}$, are further etched down from n-type $\mathrm{GaN}$ layer to the sapphire substrate through a second ICP etch. Each $\mu$ LED element is at the centre of its corresponding $\mathrm{GaN}$ mesa to guarantee a uniform current spreading. The spacing between $\mathrm{GaN}$ mesas is set as $70 \mu \mathrm{m}$ to reduce light absorption by neighbouring $\mu$ LED element and, thus, improve the optical power output of the array [21]. After these etching steps, a $100 \mathrm{~nm} \mathrm{Pd}$ metal layer is evaporated on the p-type $\mathrm{GaN}$ surface, followed a thermal annealing in a $\mathrm{N}_{2}$ ambient at $300{ }^{\circ} \mathrm{C}$ to form a quasi-ohmic contact to p-type GaN. The n-type metallization on $\mathrm{GaN}$ mesas is formed by $\mathrm{Ti} / \mathrm{Au}$ $(50 \mathrm{~nm} / 200 \mathrm{~nm})$ metal bilayer. After a plasma cleaning step by an $\mathrm{O}_{2}$ plasma at $200{ }^{\circ} \mathrm{C}$ in plasma asher, a $300 \mathrm{~nm}$-thick $\mathrm{SiO}_{2}$ layer is deposited by plasma enhanced chemical vapour deposition as an insulating layer. Then, the $\mathrm{SiO}_{2}$ on top of each $\mu \mathrm{LED}$ element and selected area on GaN mesas is removed for further metal interconnection. Finally, another Ti/Au metal bilayer is sputtered to interconnect $\mu$ LED elements following series-connection configuration. In this optimised fabrication process, we employed a plasma ashing step to minimize the fabrication errors leading to the short-circuit issue [20]. Meanwhile, in order to eliminate the fab errors causing the open-circuit issue, the metal sputter step was employed to guarantee the conform metal deposition for $\mu$ LED element interconnection. Fig. 1(b) is an optical image of the seriesbiased $\mu$ LED array at a DC operating current density of $79.6 \mathrm{~A} / \mathrm{cm}^{2}$, corresponding to $1 \mathrm{~mA}$ operating current.

\section{B. Electrical, Optical and Modulation Performance}

Fig. 2(a) shows the current density versus voltage $(J-V)$ and optical power versus current density $(L-J)$ characteristics of the series-biased $\mu \mathrm{LED}$ array fabricated in this work. These characteristics were measured at the same time by placing a $\mathrm{Si}$ photodetector in close proximity to the polished sapphire substrate of the array, through scanning each data point under DC conditions. As shown, the turn-on voltage at $79.6 \mathrm{~A} / \mathrm{cm}^{2}$ ( $1 \mathrm{~mA}$ ) of this array is $26.5 \mathrm{~V}$, corresponding to about $2.94 \mathrm{~V}$ for each $\mu \mathrm{LED}$ element. This value is consistent with the theoretical turn-on voltage of blue-emitting LEDs, which is around $2.76 \mathrm{~V}$, demonstrating a high-quality fabrication process we developed in this work. Meanwhile, this seriesbiased $\mu$ LED array can be operated at a current density up to $3200 \mathrm{~A} / \mathrm{cm}^{2}$, and is able to produce an optical power over $18.0 \mathrm{~mW}$ before thermal rollover. Moreover, as the earlier 


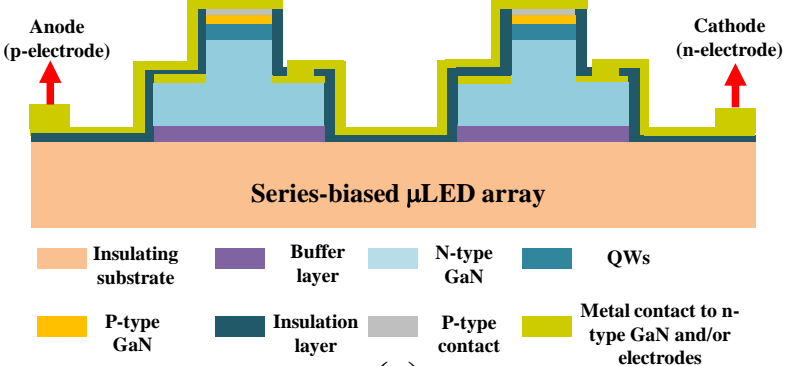

(a)

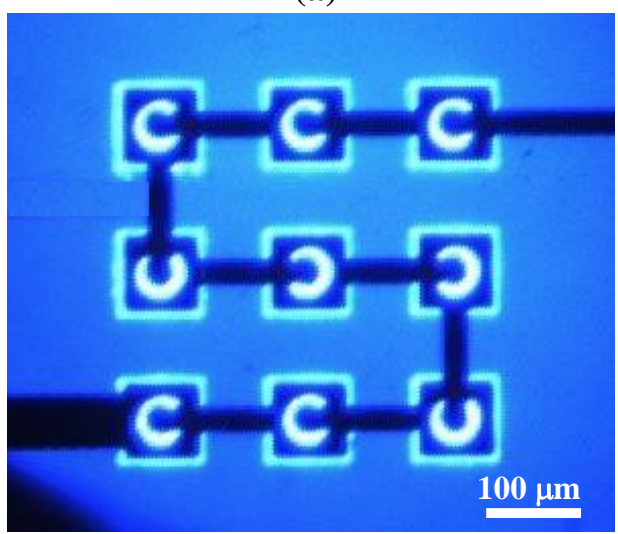

(b)

Fig. 1. (a) Simplified cross-sectional structure of a series-biased $\mu$ LED array, taking two adjacent $\mu$ LED elements as an example. Relative dimensions are not to scale. Part (b) shows an optical image of the operating array at a DC operating current density of $79.6 \mathrm{~A} / \mathrm{cm}^{2}$, corresponding to $1 \mathrm{~mA}$ operating current.

work based on a single $\mu$ LED emitter has shown [8], [9], this high operating current density leads to a shorter differential carrier lifetime and, thus, increases the modulation bandwidth. As illustrated in Fig. 2(b), the 6-dB electrical bandwidth of this array is in excess of $280 \mathrm{MHz}$ at $3200 \mathrm{~A} / \mathrm{cm}^{2}$ operating current density. These 6-dB electrical modulation bandwidths were measured following the same method described in [8] and [22]. The series-biased $\mu$ LED array was directly probed by a high-speed micro-probe and the input signal consists of a DC bias from a power supply combined with a small modulation voltage (few $\mathrm{mV}$ ) from a network analyser. The modulated light was then received by a photodiode with a $3-\mathrm{dB}$ electrical bandwidth of $1.4 \mathrm{GHz}$ and sent to the network analyser. The optical power of this series-biased LED array consisting of 9 $\mu$ LED elements is slightly lower than 9 times the optical power produced by a single $\mu$ LED element with the similar size at the same operating current density as shown in [10]. This is mainly due to light absorption by neighbouring $\mu$ LED elements [21] and heating effects in the series-biased $\mu$ LED array. On the other hand, the modulation bandwidth of this array is even higher than the one of the single $\mu$ LED element at the same operating current density. The observed higher modulation bandwidth of the series-biased $\mu$ LED array is attributed to its lower capacitance due to the series-connection configuration, which is consistent with the one reported in [19]. We are currently working on the performance comparison between the single $\mu$ LED elements, series-biased $\mu$ LED array, parallelbiased $\mu$ LED array and broad LEDs to systemically investigate
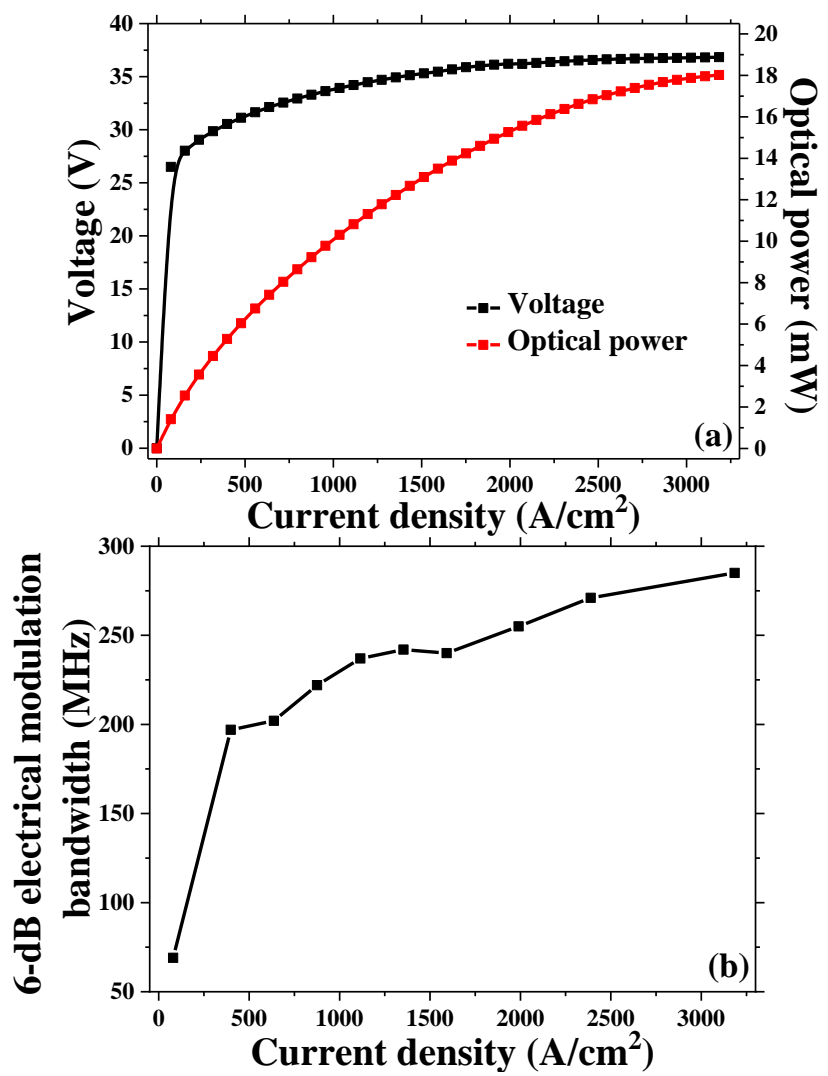

Fig. 2. (a) $J-V$ and $L-J$ characteristics of the series-biased $\mu$ LED array; (b) $6-\mathrm{dB}$ electrical bandwidth characteristic of the same series-biased $\mu$ LED array.

the advantages of the series-biased $\mu$ LED array for VLC applications and underlying physical mechanisms. Here, we also wish to emphasise that all the measurements of this seriesbiased LED array were performed on a bare chip without any thermal management. The maximum operating current density of the series-biased $\mu$ LED array is strongly limited by its selfheating effect. Thus the results shown here only demonstrate the basic capability of series-biased LED arrays in terms of high optical power output and modulation bandwidth. Even higher values on both optical power and modulation bandwidth are expected when a heat sink is applied on the array.

\section{VLC APPLICATIONS}

In this section, we present free-space data transmission results using the series-biased $\mu$ LED array as a light source. The series-biased $\mu$ LED array was modulated assuming OOK, 4-PAM and OFDM modulation formats. The results shown here aim to demonstrate the capability of series-biased $\mu \mathrm{LED}$ arrays in VLC applications.

\section{A. Experimental Setup}

The experimental setup for the free-space VLC data communication used in this work was similar to that previously reported in [11]. Different waveforms generated in MATLAB ${ }^{\circledR}$ were supplied to an arbitrary waveform generator (AWG), which mapped them to analog signals. Keysight $81180 \mathrm{~B}$ was used for OOK and PAM formats, and Keysight M8190A was 
for the OFDM formats. The output of the AWG was amplified with a high-power amplifier, SHF S126A, and a DC current density was added to the drive signal using a bias-T, Tektronix PSPL5575A. After extensive experiments to optimise the system performance, the modulation signal depths, $V_{P P}$, were set as $6.19 \mathrm{~V}$ for OOK and PAM, and $5.63 \mathrm{~V}$ for OFDM. The DC-bias current density, $J_{D C}$, was $1592.4 \mathrm{~A} / \mathrm{cm}^{2}$ for OOK and PAM, and $1074.8 \mathrm{~A} / \mathrm{cm}^{2}$ for the OFDM. The bias signal was applied to the series-biased $\mu \mathrm{LED}$ array using a highspeed micro-probe. Light emitted from the series-biased LED array was imaged onto a high-speed photodetector, Newport 1601FS-AC (3-dB electrical bandwidth of $1 \mathrm{GHz}$ ), using a high numerical aperture microscope objective, Newport M$40 \times$. The distance between the series-biased $\mu$ LED array and photo receiver was $0.3 \mathrm{~m}$. The output signal of the photodetector was captured by a digital oscilloscope, MSO7104B for OOK and PAM, and DSA90804A for OFDM.

\section{B. Free-space VLC Performance}

For a general $M$-PAM modulation format with $M$ constellation size and number of bits $k=\log _{2} M$, a set of $2 k$ possible information symbols is mapped to a set of amplitudes $\left\{A_{m}\right.$, $1 \leq m \leq M\}$, where $A_{m}=2 m-1-M$ [23]. The set of amplitudes $\left\{A_{m}\right\}$ is referred to as transmitted symbols. During the experiment, the non-return-to-zero symbols are used for the OOK modulation format, which is equivalent to the 2-PAM. The sets of OOK and 4-PAM symbols are $\{-1,1\}$ and $\{-3,-1,1,3\}$, respectively. Fig. 3 compares the eye diagrams of transmitted (a) and received (b) signals assuming the OOK modulation format at $700 \mathrm{Mbps}$ using the fabricated series-biased $\mu$ LED array. As shown, even at this relative low data rate using a simple modulation format, the amplitude and temporal distortions caused by the communication channel can be observed (Fig. 3(b)). These distortions lead to intersymbol interference (ISI) [23], [24] and a higher bit error ratio (BER). In order to mitigate the ISI, a feedforward equalizer is employed. Specifically, a finite impulse response filter whose system function in $\mathrm{z}-$ domain is denoted as $H(z)=\sum_{l=0}^{L} b_{l} z^{-l}$ is used, where $b_{l}$ is the filter coefficient and $L$ is the number of filter taps. The number of used filter taps is set to $L=9$ in this work which strikes a reasonable trade-off between computational complexity and performance enhancements given the line-ofsight channel, which is dominated by the direct, non-reflected signal component. To demonstrate the working mechanism of this equalizer, the histograms of transmitted and received symbols assuming OOK (Fig. 4(a)) and 4-PAM (Fig. 4(b)) modulation formats at $2.1 \mathrm{Gbps}$ are shown. Note that, uniformly distributed symbols are generated at the transmitter. However, the distribution of received symbols before equalizer is heavier in the negative side, which is mainly due to the ISI as discussed [23], [24]. The employed equalizer alleviates this phenomenon by estimating the received symbols. Generally, if the received symbol goes beyond a decision threshold, the estimated symbol from the equalizer will be decoded as its nearest neighbours. It leads to a narrower spread of received symbols and, therefore, the lower BER.

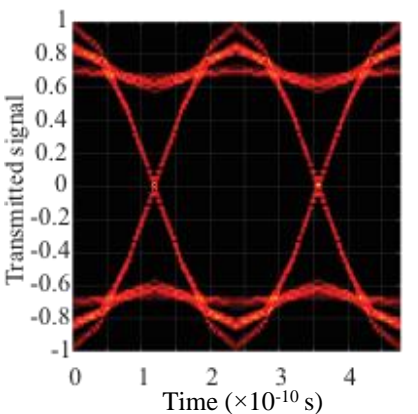

(a)

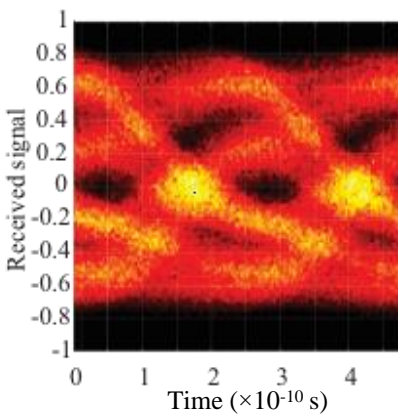

(b)
Fig. 3. Eye diagrams of (a) transmitted and (b) received signals assuming OOK modulation schemes at $700 \mathrm{Mbps}$ without equalizer using the fabricated series-biased $\mu$ LED array.

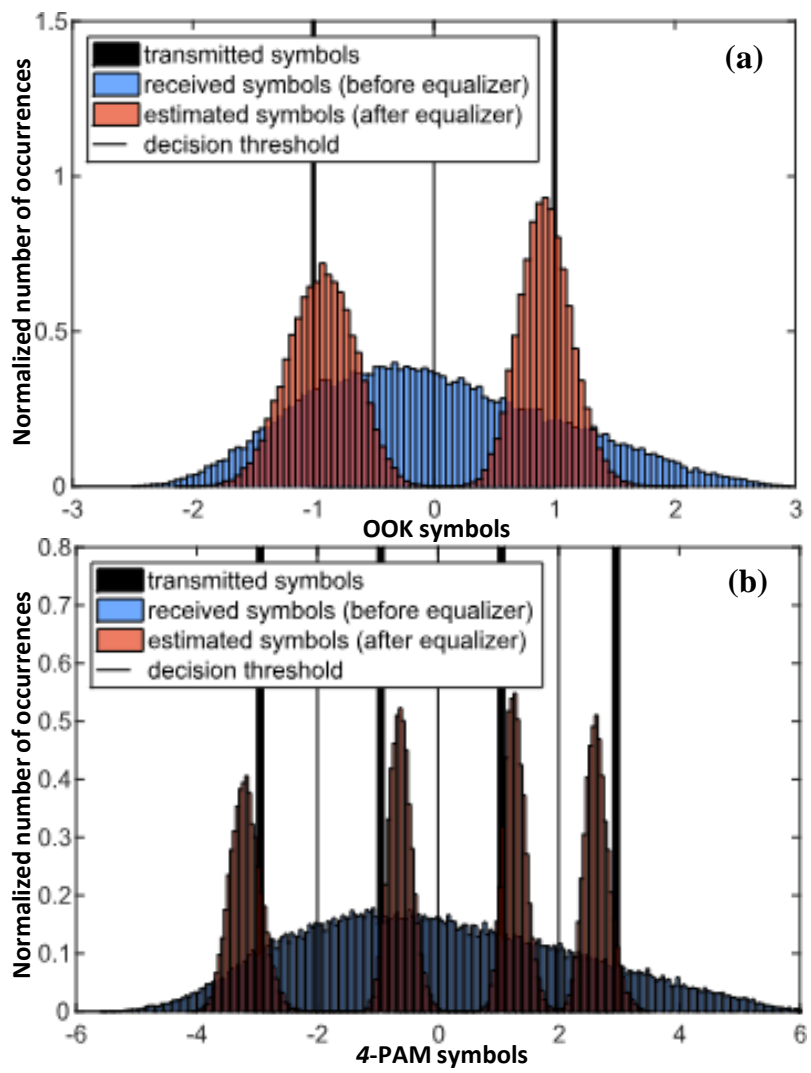

Fig. 4. Histograms of transmitted and received symbols assuming (a) OOK and (b) 4-PAM modulation formats at $2.1 \mathrm{Gbps}$ using the fabricated seriesbiased $\mu$ LED array.

A drawback of single carrier modulation such as $M$-PAM is the computational complexity of the equalizer. A cost and computational effective solution is to use OFDM with a single tap equalizer in conjunction with a cyclic prefix which is at least as long as the largest multi-path component assumed to be between 3-10 ns. The number of subcarriers and hence the OFDM frame length will be chosen so that the spectrum efficiency loss is less than $5 \%$. Binary bits are modulated into $\mathrm{M}$-ary quadrature amplitude modulation ( $M$-QAM) symbols. Adaptive bit and energy loading is used to allow different constellation sizes to be loaded on the subcarriers based on 


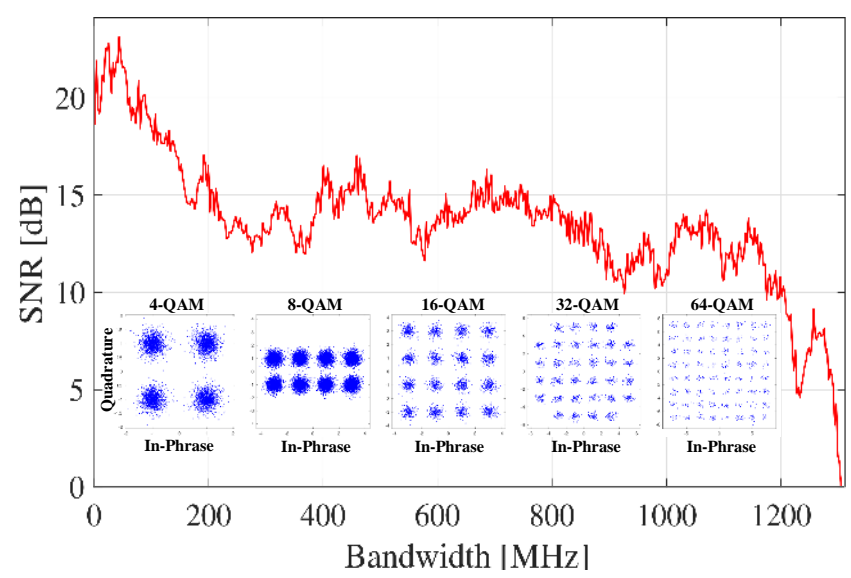

Fig. 5. SNR versus bandwidth achieved for OFDM using the fabricated seriesbiased $\mu \mathrm{LED}$ array at $J_{D C}=1074.8 \mathrm{~A} / \mathrm{cm}^{2}$ and $V_{P P}=5.63 \mathrm{~V}$. The inserts are the $M$-QAM constellation symbols received at the photodetector after equalization for $M=4,8,16,32,64$.

the available SNR. This is done in consistency with our previous work [11]. A frame of $N_{F F T} / 2-1$ QAM symbols is then multiplexed into NFFT subcarriers using inverse Fourier transformation (IFFT). Hermitian symmetry is imposed on the OFDM frame to guarantee a real-valued output. A DC bias is used to shift the negative OFDM samples into positive. The generated digital waveform is converted into analog by the AWG at a sampling frequency of $10.8 \mathrm{GSps}$. Root raised cosine is used with an oversampling factor of 4 , which corresponds to a single-sided modulation bandwidth of $1.35 \mathrm{GHz}$. The fast Fourier transformation (FFT) is applied on the received signal and the received QAM symbols are equalized using the estimated channel. By using the fabricated seriesbiased $\mu \mathrm{LED}$ array, the recovered $M$-QAM constellations for $M=4,8,16,32,64$ are shown as the inserts of Fig. 5. They demonstrate that the SNR is high enough to allow maximum likelihood decoder to distinguish the transmitted symbols. The SNR versus bandwidth of the series-biased $\mu$ LED array at $J_{D C}=1074.8 \mathrm{~A} / \mathrm{cm}^{2}$ and $V_{P P}=5.63 \mathrm{~V}$ is shown in Fig. 5. The series-biased $\mu$ LED array has shown an impressive SNR performance that is higher than $10 \mathrm{~dB}$ up to $1.2 \mathrm{GHz}$ of bandwidth. This permits high speed data communications to be demonstrated.

Fig. 6 summarises the measured data transmission rates using the fabricated series-biased $\mu$ LED array as the transmitter assuming OOK, 4-PAM and OFDM modulation formats. As shown, the maximum data rates at the FEC floor of $3.8 \times 10^{-3}$ are $2.1,2.55$, and $5.18 \mathrm{Gbps}$ for OOK, 4-PAM and OFDM with adaptive modulation formats, respectively. After applying a $7 \%$ FEC overhead reduction, the error-free date rates of $1.95,2.37$ and $4.81 \mathrm{Gbps}$ are achieved. To the best of our knowledge, this is the first work presenting the VLC performances using a series-biased $\mu$ LED array as the transmitter. Compared with our previous work using a single $\mu$ LED element as the transmitter [10], [11], these values are slightly lower. The pronounced self-heating effect of seriesbiased $\mu \mathrm{LED}$ arrays is considered as the main factor limiting the data rates we can achieved. Firstly, as we demonstrated in

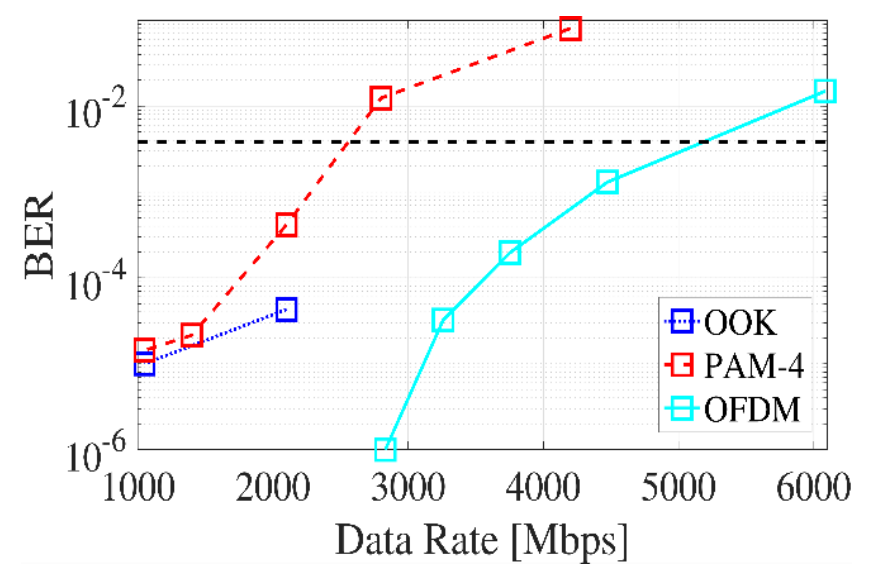

Fig. 6. BER versus data rate achieved using the fabricated series-biased $\mu$ LED array as the transmitter assuming OOK, 4-PAM and OFDM modulation formats, respectively.

section II B, this effect imposes a restriction on the operating current density of the arrays. This leads to a lower $J_{D C}$ applied on the array and, thus, a lower modulation bandwidth used in data communication measurements. Secondly, the linear region of the optical power versus voltage characteristic of the array are also narrowed by the self-heating effect. This results in a relative shallow $V_{P P}$ set in the measurement, which degrades the SNR. Currently, we are working on applying a custom heat sink to the series-biased $\mu \mathrm{LED}$ arrays to alleviate this effect and, in turn, achieve higher data rates.

\section{CONCLUSiON}

We presented the design, fabrication and performance characterisation of III-nitride series-biased $\mu$ LED arrays for VLC applications. An example array, which consists of $3 \times 340 \mu \mathrm{m}-$ in-diameter $\mu \mathrm{LED}$ elements, was fabricated from a commercial blue LED wafer on sapphire substrate. This series-biased $\mu$ LED array shows the outstanding electrical, optical and modulation performance. At an operating current density of $3200 \mathrm{~A} / \mathrm{cm}^{2}$, over $18.0 \mathrm{~mW}$ optical power and a 6-dB electrical bandwidth of $285 \mathrm{MHz}$ are achieved. We further demonstrated the free-space VLC application of this series-biased $\mu$ LED array assuming OOK, PAM and OFDM modulation formats and achieved the error-free data rates of 1.95, 2.37 and $4.81 \mathrm{Gbps}$, respectively. All the data achieved in this work were measured from a bare chip and, thus, strongly limited by the self-heating effect from series-biased $\mu$ LED arrays. We believe that, by integrating the array with heat sinks, the data rate would be further improved for both short- and longdistance VLC applications.

\section{REFERENCES}

[1] H. Burchardt, N. Serafimovski, D. Tsonev, S. Videv, and H. Haas, "VLC: Beyond point-to-point communication." IEEE Communications Magazine, vol. 52, no. 7, pp. 98-105, 2014.

[2] J. Grubor, S. C. J. Lee, K.-D. Langer, T. Koonen, and J. W. Walewski, "Wireless high-speed data transmission with phosphorescent white-light LEDs," in Optical Communication-Post-Deadline Papers (published 2008), 2007 33rd European Conference and Exhibition of. VDE, 2007, pp. $1-2$. 
[3] A. Khalid, G. Cossu, R. Corsini, P. Choudhury, and E. Ciaramella, "1-Gb/s transmission over a phosphorescent white LED by using rateadaptive discrete multitone modulation," IEEE Photonics Journal, vol. 4, no. 5, pp. 1465-1473, 2012.

[4] A. H. Azhar, T. Tran, and D. OBrien, "A gigabit/s indoor wireless transmission using MIMO-OFDM visible-light communications," IEEE photonics technology letters, vol. 25, no. 2, pp. 171-174, 2013.

[5] Y. Zhou, J. Zhao, M. Zhang, J. Shi, and N. Chi, "2.32 Gbit/s phosphorescent white LED visible light communication aided by two-staged linear software equalizer," in Communication Systems, Networks and Digital Signal Processing (CSNDSP), 2016 10th International Symposium on. IEEE, 2016, pp. 1-4.

[6] J.-W. Shi, H.-Y. Huang, J.-K. Sheu, C.-H. Chen, Y.-S. Wu, and W.-C. Lai, "The improvement in modulation speed of GaN-based Green lightemitting diode (LED) by use of n-type barrier doping for plastic optical fiber (POF) communication," IEEE photonics technology letters, vol. 18 , no. 15 , pp. $1636-1638,2006$.

[7] C.-L. Liao, Y.-F. Chang, C.-L. Ho, and M.-C. Wu, "High-speed GaNbased blue light-emitting diodes with gallium-doped $\mathrm{ZnO}$ current spreading layer," IEEE Electron Device Letters, vol. 34, no. 5, pp. 611-613, 2013.

[8] J. J. McKendry, D. Massoubre, S. Zhang, B. R. Rae, R. P. Green, E. Gu, R. K. Henderson, A. Kelly, and M. D. Dawson, "Visible-light communications using a CMOS-controlled micro-light-emitting-diode array," Journal of lightwave technology, vol. 30, no. 1, pp. 61-67, 2012.

[9] J. J. McKendry, R. P. Green, A. Kelly, Z. Gong, B. Guilhabert, D. Massoubre, E. Gu, and M. D. Dawson, "High-speed visible light communications using individual pixels in a micro light-emitting diode array," IEEE Photonics Technology Letters, vol. 22, no. 18, pp. 1346$1348,2010$.

[10] R. X. Ferreira, E. Xie, J. J. McKendry, S. Rajbhandari, H. Chun, G. Faulkner, S. Watson, A. E. Kelly, E. Gu, R. V. Penty et al., "High bandwidth GaN-based micro-LEDs for multi-Gb/s visible light communications," IEEE Photonics Technology Letters, vol. 28, no. 19, pp. 2023-2026, 2016.

[11] M. S. Islim, R. X. Ferreira, X. He, E. Xie, S. Videv, S. Viola, S. Watson, N. Bamiedakis, R. V. Penty, I. H. White et al., "Towards $10 \mathrm{~Gb} / \mathrm{s}$ orthogonal frequency division multiplexing-based visible light communication using a GaN violet micro-LED," Photonics Research, vol. 5, no. 2, pp. A35-A43, 2017.

[12] C. E. Shannon, "A mathematical theory of communication," $A C M$ SIGMOBILE mobile computing and communications review, vol. 5, no. 1 , pp. 3-55, 2001

[13] S. Rajbhandari, H. Chun, G. E. Faulkner, K. L. Cameron, A. V. Jalajakumari, R. K. Henderson, D. Tsonev, M. Ijaz, Z. Chen, H. Haas et al., "High-Speed Integrated Visible Light Communication System: Device Constraints and Design Considerations." IEEE Journal on Selected Areas in Communications, vol. 33, no. 9, pp. 1750-1757, 2015

[14] C.-H. Tien, K.-Y. Chen, C.-P. Hsu, and R.-H. Horng, "Enhanced light output power of thin film GaN-based high voltage light-emitting diodes," Optics express, vol. 22, no. 106, pp. A1462-A1468, 2014.

[15] S. Li, K.-T. Lam, W.-C. Huang, and S.-J. Chang, "Effects of microcell layout on the performance of GaN-based high-voltage light-emitting diodes," Journal of Photonics for Energy, vol. 5, no. 1, p. 057605, 2015.

[16] Y. Cai, X. Zou, W. C. Chong, and K. M. Lau, "Optimization of electrode structure for flip-chip HVLED via two-level metallization," physica status solidi (a), vol. 213, no. 5, pp. 1199-1203, 2016.

[17] X. Zou, Y. Cai, W. C. Chong, and K. M. Lau, "Fabrication and characterization of high-voltage LEDs using photoresist-filled-trench technique," Journal of Display Technology, vol. 12, no. 4, pp. 397-401, 2016.

[18] C. Wang, D. Lin, C. Lee, M. Tsai, G. Chen, H. Kuo, W. Hsu, H. Kuo, T. Lu, S. Wang et al., "Efficiency and droop improvement in GaNbased high-voltage light-emitting diodes," IEEE Electron Device Letters, vol. 32, no. 8, pp. 1098-1100, 2011.

[19] Y. Huang, Z. Guo, H. Huang, and H. Sun, "Influence of current density and capacitance on the bandwidth of VLC LED," IEEE Photonics Technology Letters, vol. 30, no. 9, pp. 773-776, 2018.

[20] E. Xie, M. Stonehouse, R. Ferreira, J. J. McKendry, J. Herrnsdorf, X. He, S. Rajbhandari, H. Chun, A. V. Jalajakumari, O. Almer et al., "Design, Fabrication, and Application of GaN-Based Micro-LED Arrays With Individual Addressing by N-Electrodes," IEEE Photonics Journal, vol. 9, no. 6 , pp. $1-11,2017$.

[21] C.-H. Tien, C.-H. Kuo, D.-S. Wuu, and R.-H. Horng, "Improved Optoelectronic Performance of High-Voltage Ultraviolet Light-Emitting Diodes Through Electrode Designs," IEEE Transactions on Electron Devices, vol. 64, no. 11, pp. 4526-4531, 2017.
[22] J. J. McKendry, B. R. Rae, Z. Gong, K. R. Muir, B. Guilhabert, D. Massoubre, E. Gu, D. Renshaw, M. D. Dawson, and R. K. Henderson, "Individually addressable AlInGaN micro-led arrays with CMOS control and subnanosecond output pulses," IEEE Photonics Technology Letters, vol. 21, no. 12, pp. 811-813, 2009.

[23] J. G. Proakis and M. Salehi, Digital communications. McGraw-Hill, 2008.

[24] J. K. Perin, M. Sharif, and J. M. Kahn, "Sensitivity improvement in $100 \mathrm{~Gb} / \mathrm{s}$-per-wavelength links using semiconductor optical amplifiers or avalanche photodiodes," Journal of Lightwave Technology, vol. 34, no. 23 , pp. $5542-5553,2016$

Enyuan Xie received the Ph.D. degree in physics from the University of Strathclyde, Glasgow, U.K., in 2013. Since then, he has been with the Institute of Photonics, University of Strathclyde, Glasgow, U.K., as a Research Associate, becoming involved in the fabrication, characterization, and application of GaN-based micro-LED arrays.

Xiangyu He received the M.Sc. degree in photonics and device microfabrication from University of Strathclyde, Glasgow, U.K., in 2015. He is currently working toward the Ph.D. degree at the Institute of Photonics, University of Strathclyde. His research interests include micro-LED fabrication and optical communications.

Mohamed Sufyan Islim (S'07) received the M.Sc. degree (distinction) in communications engineering from Aleppo University, Aleppo, Syria, in 2013, and the M.Sc. degree (distinction) in signal processing and communications, from the University of Edinburgh, Edinburgh, U.K., in 2014. He is currently working toward the Ph.D. degree at the LiFi Research and Development Centre, University of Edinburgh. His research interests include optical OFDM, $\mathrm{LiFi}$, and optical wireless communications. Among several scholarships he was awarded in 2013, he was the recipient of the Global Edinburgh Syrian Scholarship from Edinburgh University. He also received the 2014 IEEE communications chapter prize for the best master project.

Ardimas Andi Purwita (S'15) received the B.S. and M.Sc. degrees in electrical engineering from the Institut Teknologi Bandung, Bandung, Indonesia, in 2011 and 2014, respectively. He is currently pursuing the Ph.D. degree with The University of Edinburgh, Edinburgh, U.K. He was with the School of Information Science, Japan Advanced Institute of Science and Technology in 2015 as a researcher. His current research interests include channel coding, interference mitigation, and resource allocation.

Jonathan J. D. McKendry received the M.S. degree in electronics and electrical engineering from the University of Glasgow, Glasgow, U.K., in 2006, and the Ph.D. degree from the University of Strathclyde, Glasgow, U.K., in 2011. In 2007, he joined the Institute of Photonics as a Ph.D. student where the focus of his Ph.D. was on the application of AlInGaNbased micro-LEDs for time-resolved fluorescence lifetime measurements and optical communications. He currently works as a Research Associate with the Institute of Photonics, University of Strathclyde, Glasgow, U.K., primarily on the subject of LED-based visible light communications. To date, he has authored or co-authored more than 30 peer-eviewed journal articles and 30 conference submissions. 
Erdan Gu received the Ph.D. degree in thin film physics from Aberdeen University, U.K., in 1992. Afterward, he was a Research Fellow with the Cavendish Laboratory, Cambridge University, Cambridge, U.K. In 1997, he joined the Thin Film Group, Oxford Instruments plc, U.K. as a Senior Research Scientist working on superconducting photonic devices. Since July 2002, he has been with the Institute of Photonics, University of Strathclyde, Glasgow, U.K., where he is an Associate Director and a Research Team Leader. In the Institute of Photonics, he is working and playing a leading role in a range of research projects on photonic materials and devices, micro/nano optoelectronics, diamond photonics, and optoelectronic devices for visible light communications.

Harald Haas (S'98-AM'00-M'03-SM'17-F'18) received the Ph.D. degree from the University of Edinburgh, Edinburgh, U.K., in 2001. He is currently the Chair of Mobile Communications, University of Edinburgh, and the Founder and Chief Scientific Officer of pureLiFi Ltd. pureLiFi Ltd has more than 50 employees and has operations in the USA and Singapore. $\mathrm{He}$ is also the Director of the LiFi Research and Development Center, University of Edinburgh. His main research interests include optical wireless communications, hybrid optical wireless and RF communications, spatial modulation, and interference coordination in wireless networks. He first introduced and coined spatial modulation and LiFi. LiFi was listed among the 50 best inventions in TIME Magazine 2011. He was an invited speaker at TED Global 2011, and his talk: Wireless Data from Every Light Bulb has been watched online more than 2.5 million times. He gave a second TED Global lecture in 2015 on the use of solar cells as LiFi data detectors and energy harvesters. This has been viewed online more than 2.0 million times. He holds 43 patents and has more than 30 pending patent applications. He has published more than 400 conference and journal papers including a paper in Science. His Google Scholar h-index is 67, and his works have been cited more than 19,000 times. He was on the Thomson Reuters list of highly cited scientists, 2017. He co-authors a book entitled: Principles of LED Light Communications Towards Networked Li-Fi (Cambridge University Press, Cambridge, U.K., 2015).

Prof. Haas is an editor for the IEEE TRANSACTIONS ON COMMUNICATIONS and the IEEE JOURNAL OF LIGHTWAVE TECHNOLOGY. He was the co-recipient of the recent Best Paper Awards at VTC-Fall, 2013, VTC-Spring 2015, ICC 2016, and ICC 2017. He was the co-recipient of the EURASIP Best Paper Award for the Journal on Wireless Communications and Networking in 2015 and co-recipient of the Jack Neubauer Memorial Award of the IEEE Vehicular Technology Society. He is the recipient of the Tam Dalyell Prize 2013 awarded by the University of Edinburgh for excellence in engaging the public with science. In 2016, he was the recipient of the Outstanding Achievement Award from the International Solid State Lighting Alliance, which was awarded to him by Prof. Shuji Nakamura. He has delivered 50 keynote talks at international conferences and workshops. In 2012, he was the recipient of the prestigious Established Career Fellowship from the Engineering and Physical Sciences Research Council within information and communications technology in the U.K. In 2014, he was selected by EPSRC as one of ten recognising inspirational scientists and engineers (RISE) Leaders in the U.K. He was elected a Fellow of the Royal Society of Edinburgh in 2017.

Martin D. Dawson (M'85-SM'98-F'09) is a physicist known for his work on lasers and compound semiconductors. He is the Director of Research with the Institute of Photonics, University of Strathclyde, Glasgow, U.K., which he helped establish 20 years ago, and he was also appointed Inaugural Head of the Fraunhofer Centre for Applied Photonics in October 2012. He has more than 30 years' experience of applied research gained in academia and industry in the U.K. and USA, and he has been involved in the formation and technical development of a number of spin-out businesses, most recently including mLED Ltd. He holds an EPSRC Programme Grant on visible light communications and gave a Rank Prize Lecture in 2014 on applied research in photonics. He was the recipient of the 2016 Gabor Medal and Prize by the Institute of Physics and the 2016 Aron Kessel Award by the IEEE Photonics Society. 